
\title{
25 Research Square \\ Comparative Study OCT versus MRI T2 in Diagnosis of Degenerative Cartilage Lesions
}

\section{Cosmin loan Faur}

Universitatea de Medicina si Farmacie Victor Babes din Timisoara

Ahmed Abu-Awwad ( $\nabla$ ahm.abuawwad@umft.ro )

Universitatea de Medicina si Farmacie Victor Babes din Timisoara

Mariana Tudoran

Universitatea de Medicina si Farmacie Victor Babes din Timisoara

Ana Maria Ungureanu

Universitatea de Medicina si Farmacie Victor Babes din Timisoara

Cristina Tudoran

Universitatea de Medicina si Farmacie Victor Babes din Timisoara

Jenel Marian Patrascu

Universitatea de Medicina si Farmacie Victor Babes din Timisoara

Cosmin Sinescu

Universitatea de Medicina si Farmacie Victor Babes din Timisoara

\section{Research article}

Keywords: Optical Coherence Tomography; MRl; arthroscopic examination; degenerative cartilage defects

Posted Date: May 29th, 2020

DOI: https://doi.org/10.21203/rs.3.rs-29937/v1

License: (c) (i) This work is licensed under a Creative Commons Attribution 4.0 International License.

Read Full License 


\section{Abstract}

\section{Background}

When diagnosed early, degenerative cartilage lesions are reversible if the appropriate therapeutical measures are applied. Currently available diagnostic methods (radiography, MRI and arthroscopic examination) do not provide the accuracy needed in the early diagnosis of these lesions.

\section{Methods}

The aim of the study is to analyse, in an ex vivo study on osteochondral fragments taken from patients undergoing total knee arthroplasty, weather the OCT technique is effective in detecting these lesions. The results will be compared to the preoperative MRI images (especially $T_{2}$ mapping technique).

Results

The percentage of the agreement between the two reviewers was $89 \%$ (8 out of 9 cases) during the OCT examination process.

\section{Conclusion}

OCT is a new technique potentially useful in the diagnosis of cartilage lesions. The possibility of performing it through minimally invasive techniques currently (arthroscopic surgery) further increases the value of this technique. OCT is a non-destructive diagnosing technique in degenerative cartilage lesions whose efficiency is comparable to MRI T2 technique and superior to conventional MRI.

\section{Background}

Osteoarthritis is a joint morbidity with high incidence in the general population which affects especially the older patients. This condition involves both cartilage, ligaments, menisci, synovial membrane and the articular bone structures. However, the basic lesion is represented by cartilage degeneration.

Current treatment options in advanced stages like total knee arthroplasty are unable to provide solutions to preserve cartilage and also have limited reliability over time.

Early diagnosis of articular cartilage lesions is extremely important in order to avoid progression to advanced stages of osteoarthritis. An early diagnosis of these lesions should avoid the irreversible changes in the cartilage matrix and would facilitate the application of effective therapeutic measures for the preservation of the articular surfaces.

The radiological examination is the classical imaging diagnostic procedure used for detecting the osteoarthritic changes. Performed in two standard views (postero-anterior and lateral) this investigation highlights the two characteristic changes of osteoarthritis: the presence of osteophytes and joint space narrowing. These changes are classified according to Kellgren's criteria [1]. 
Another imaging method used to assess changes in osteoarthritic cartilage is MRI. Conventional MRI can provide data on cartilage lesions in advanced stages with partial or full thickness discontinuities of the articular surface but is not able to distinguish between healthy cartilage and early degenerative changes when the articular surface is intact (subsurface lesions) [2]. New MRI techniques are developing, techniques capable to provide useful information on the morphology or physiology of the in the early stages of degeneration of the collagen matrix: MRI T2 mapping, three -dimensional spoiled gradient recalled echo imaging with fat suppression (3D - SPGR), sodium-MRI or delayed Gadolinium-enhanced MRI (dGEMRIC) [3]. MRI T2 mapping technique detects changes in orientation of fibers and hydration of collagen layer. With these properties this technique may provide details of structural integrity of the cartilage with intact articular surface as well as regarding biochemical integrity of the cartilage [4]. Among the disadvantages of this technique we mention the impossibility of determining the stage of the lesion [5] as well as the fact that the level of physical activity may influence the results [6].

Currently arthroscopic examination represents the golden standard in the diagnosis of degenerative cartilage changes. Outerbridge classification is used for assessment of the stage articular lesions. The major disadvantage of this technique, in addition to being an invasive technique, is the fact that the diagnosis of cartilage injury is established through a combination of imaging techniques to explore the articular surface combined with the tactile sense of the examiner used to check the roughness of the joint surface. This means that arthroscopy cannot detect subsurface cartilage lesions until they affect articular surface and usually in this stage the changes are irreversible.

New techniques capable to detect structural changes of the collagen matrix of the cartilage with intact surface are needed, making possible to apply an early treatment with the final aim: preservation of the articular surface. Optical Coherence Tomography (OCT) is a new diagnostic method that uses the infrared rays that can detect traumatic or degenerative changes occurring in the collagen layers of articular cartilage [7]. The advantages of OCT technique are small portable device with the possibility of incorporating technology in arthroscopic technique, the resolution nearly 25 times greater than any medical technology [8], data acquisition rate of 120 frames per second. Another advantage of this technique is that associated with spectroscopic techniques (PS-OCT) it can measure the collagen content of the desired anatomical specimen $[9,10]$ OCT can analyze sections of articular cartilage in a scale of 10 to 20 microns comparable to histopathologic examination [11-13].

The scope of this paper is to assess the utility of OCT technology in early detection of degenerative cartilage lesions of the knee joint through a comparative study with MRI T2 mapping technique.

\section{Methods}

In this study 9 patients were examined. All patients in the study group had undergone bi or tricompartimental knee arthroplasties. Criteria for inclusion in the study were the absence of arthritic changes in the external femoro-tibial compartment on the preoperative radiological examination 
(Kellgren-Lawrence stage 0 and 1). The mean age of patients was 56.1 years (range between 52 and 61 years), the ratio female: male was 6:3.

All patients selected in the study group performed a MRI investigation, for maximum 21 days preoperatively. The MRI studies were performed with a clinical 1.5T MRI system with Tim technology (Magnetom Avanto, Siemens, Germany) using a standard extremity coil and eight-channel knee coil year. In the T2 relaxation times our Examinations were obtained from T2-maps reconstructed using a multiecho spin echo (SE) measurement with a repetition time (TR) of $4130 \mathrm{~ms}$ and echo times (TE) between $10 \mathrm{~ms}$ (minimum) and $87 \mathrm{~ms}$ (maximal value). Field of View (FoV) WAS 200x200mm, 320x320 pixel matrix and voxel size $0.6 \times 0.6 \times 3.0 \mathrm{~mm}$. All of the images Were Prepared Within the syngoMaplt using Inline Technology. These post-processed using maps Were syngoFusion to overlay maps with Their Corresponding These anatomical images and to conduct manual cartilage segmentation (Figure 1A, B).

The interpretation of the acquired MRI data was performed by two independent observers after a prior randomization of the patients. The study area included an area of $4 \mathrm{~mm}$ diameter from the center of the lateral tibial plateau. Measurements were recorded differently depending on the depth of the examined cartilage. The thickness of the tibial plateau cartilage was divided into two halves: deep and superficial, the average values for the two areas were recorded. For the comparative analysis OCT versus MRI T2mapping technique were used only the values (in $\mathrm{msec}$ ), obtained in the superficial half of the cartilage layer.

The surgical procedure was performed for all patients in the $2^{\text {nd }}$ Department of Orthopedics and Traumatology of the Emergency County Hospital Timisoara. In all cases we used ACGII system (Biomet, Warsaw, Indiana). Seven of the patients received a tricompartmental knee endoprostheses the other two underwent a bicompartmental knee arthroplasty (arthroplasty of the medial and lateral tibio-femoral compartments). During tibial plateau osteotomy, attention was paid in order to extract enblock the whole tibial articular surface, avoiding damage of the articular cartilage especially in the lateral tibio-femoral compartment (Figure 2).

The tibial articular fragment containing the lateral and medial tibial plateaus was examined with the OCT technique. This examination was performed only for the lateral tibial plateau within 3 hours postoperatively after a standardized protocol.

A Time Domain (TD) Optical Coherence Tomography (OCT) system working at $1300 \mathrm{~nm}$ was used in this study (Figure 3).

The system is based on an interferometer equipped with a scanning system in three dimensions. Using these scanners, different contours, surfaces or volumes of chondral articular surfaces can be obtained, used for quality and durability. The system provides the following modes of scanning:

Dimensional outline-in depth (A-scan), which provides a variation of chondral material reflectivity in depth; 
Horizontally oriented surface (C-scan), at a certain depth that can be selected using a scanning device in subsurface; it provides a two-dimensional (2-D) distribution of chondral tissue;

Vertically oriented surface (B-scan), which provides a 2-D distribution of chondral material in a section in depth, which can be repeated at different orientations in the horizontal plane using two dual axis 2-D galvoscanners.

The size of the lateral aspect of the image, C-scan and B-scan can be adjusted continuously up to $10 \mathrm{~mm}$;

Data analysis after OCT examination was performed by two independent examiners after a prior randomization of the patients. Degenerative changes of the cartilage structure were staged using the following classification: cartilage with normal laminar structure of the collagen fibers (grade 0 ), loss of the laminar appearance of cartilage (grade 1), and cartilage with surface irregularities (grade 2) (Figure 4).

All patients agreed to participate in this study. The Emergency Hospital Timisoara leadership agreed to conduct this study.

\section{Results}

During OCT examination, the percentage of the agreement between the two reviewers was $89 \%$ (8 out of 9 cases). The sample which was the basis of a controversy between the two examiners was removed from the study (Table 1).

Table 1. Example of patients by gender, age and type of investigations.

\begin{tabular}{cccccc}
\hline Patient & Gender & Age & OCT & MRI Superficial & MRI Deep \\
\hline MA & F & 55 & 0 & 42 & 42 \\
CG & F & 57 & 1 & 49 & 41 \\
SJ & M & 61 & 1 & 52 & 42 \\
GC & F & 52 & 2 & 61 & 44 \\
UL & M & 60 & 2 & 58 & 44 \\
PA & M & 55 & 1 & 45 & 38 \\
RS & F & 54 & 1 & 44 & 41 \\
TB & F & 59 & 1 & 47 & 42 \\
\hline
\end{tabular}

For patients that remained in the study in two cases there was a normal structure with laminar aspect of the cartilage (grade 0). Five samples presented loss of characteristic laminar structure of collagen matrix, these samples were classified as grade 1, the remaining two samples presented surface irregularities of the articular surface (grade 2).

Conventional MRI technique showed in 5 cases a homogeneous appearance with uniform thickness of articular cartilage in the external tibial plateau.

After MRI T2 mapping reconstruction technique for all the patients in the study group: deep area of the examined cartilage the average value was $41.3 \mathrm{~ms}$ (range between 38 and $44 \mathrm{~ms}$ ). In the superficial area 
the average value was $25 \%$ higher $51.4 \mathrm{~ms}$ (range 42 to $61 \mathrm{~ms}$ ).

Comparative analysis of OCT versus MRI T2 mapping indicates a good correlation between OCT grading system and MRI T2 values in the superficial cartilage.

\section{Discussion}

New techniques that are capable to detect subsurface degenerative changes of the collagen matrix are needed in the early diagnosis of cartilage defects, before such changes become irreversible. Along with the ability to highlight these changes this technique should be easy to use, minimally invasive and must provide a high-quality image of the investigated anatomical structure. In this context, the studies conducted so far on the OCT technique for the early diagnosis of cartilage lesions show the efficiency of this examination.

Chu et al. (2010) [14] concluded in his study on 30 patients that OCT and MRI correlated with arthroscopic findings in early degenerative lesions.

Zheng et al. (2010) [15] in a comparative in vivo study between OCT, MRI and arthroscopy found OCT to be a potentially powerful method in early diagnosis of osteoarthritis.

Bear et al. (2009) [16] found OCT and MRI T2 to be sensitive in cartilage collagen changes in a study on anatomical specimens.

As well several other animal studies are pointing out the potential of this novel minimally invasive technique in early diagnosis of subsurface cartilage changes $[17,18]$.

This paper comes to confirm these works regarding OCT technique, and the fact that this examination method may have its well-defined place as an effective diagnostic tool for subsurface cartilage degenerative changes.

The fact that results obtained with this technique correlate with those of the T2 mapping MRI technique, the capacity of MRI T2 mapping technique to diagnose abnormalities of the collagen matrix of the cartilage has been demonstrated [19-21], in connection with the unique quality resolution images, and that the investigation can be carried out during the arthroscopic examination are all huge benefits of this technique.

Further studies should focus on an attempt to quantify and classify these lesions based on OCT data and in conjunction with medium-to long-term results regarding the evolution of the previous diagnosed cartilage lesions to be able to make correlations with available treatment options.

\section{Conclusions}


OCT is a minimally invasive diagnostic technique with comparable efficacy with MRI T2 mapping technique and superior to conventional MRI in early diagnosis of degenerative lesions of the cartilage with intact articular surface. However further quantitative studies and an objective classification of the chondral defects based on OCT images are needed.

\section{Abbreviations}

OCT - Optical Coherence Tomography

MRI - Magnetic resonance imaging

dGEMRIC - Gadolinium-enhanced magnetic resonance imaging

PS-OCT - Polarization sensitive Optical Coherence Tomography

TR - repetition time

TE - echo times

TD - Time Domain

\section{Declarations}

\section{Acknowledgements}

Prof A. Gh. Podoleanu, School of Physical Sciences, Ingram, University of Kent, Canterbury, for his assistance.

\section{Authors' contributions}

All authors (FC, AA, TM, TC, UA, PJ, SC) contributed to study conception and design. FC, PJ and SC coordinated and managed all parts of the study. UA carried out the literature search. AA conducted data collection and performed preliminary data preparations. FC, TC and TM conducted data analyses and all the authors contributed to the interpretation of data. FC wrote the first draft of the paper and all authors provided substantive feedback on the paper and contributed to the final manuscript. All authors read and approved the final manuscript.

\section{Funding}

No funding was obtained for this study.

\section{Availability of data and materials}

The datasets generated and/or analyzed during the current study are not publicly available but are available from the corresponding author on reasonable request. 


\section{Ethics approval and consent to participate}

The study was approved by the Local Ethics commission for scientific research of Emergency Clinical County Hospital Timisoara (record number 205/21.09.2018). All patients participated free-willingly and with written informed consent to the study.

\section{Consent for publication}

Not applicable.

\section{Competing interests}

The authors declare that they have no competing interests.

Authors' contributions

Conceptualization C.F. and A.A.; methodology J.M.P., A.M.U., M.T and C.T..; software, C.S., A.M.U.; validation, C.F., C.T., and J.M.P..; formal analysis, C.F..; investigation, C.F., A.A..; resources, C.F..; data curation, C.F., C.S. and J.M.P.; writing-original draft preparation, C.F., C.S. and A.A..; writing-review and editing, C.F. and A.A..; visualization, A.M.U and C.S..; supervision, C.F. and C.S..; project administration, C.F. and C.S.;

Consent for publication

Not applicable.

All authors have read and agreed to the published version of the manuscript

Funding: This research received no external funding

Conflicts of Interest: The authors declare no conflict of interest

Author details

1. Victor Babes University of Medicine and Pharmacy, 300041, Timisoara, Romania

\section{References}

1. Kallgren JH, Lawrence JS. Radiological assessment of osteo-arthrosis. Ann Rheum Dis;. 1957;16:494-502.

2. Potter HG, Linklater JM, Allen AA, Hannifin JA, Haas SB. Magnetic resonance imaging of articular cartilage in the knee: an evaluation with use of fast-spin-echo imaging. J Bone Joint Surg Am;. 1998;80:1276-84. 
3. Braun HJ, Gold GE. 2011. Diagnosis of osteoarthritis: Imaging. Bone, doi: 10.1016/j.bone.2011.11.019.

4. Potter HG, Black BR, Chong le R. New techniques in articular cartilage imaging. Clin Sports Med. 2009;28:77-94.

5. Koff MF, Amrami KK, Kaufman KR. Clinical evaluation of T2 values of patellar cartilage in patients with osteoarthritis. Osteoarthritis Cartilage Feb. 2007;15(2):198-204.

6. Stehling C, Luke A, Stahl R, Baum T, Joseph G, Pan J, Link TM. Meniscal T1rho and T2 measured with 3.0T MRI increases directly after running a marathon. Skeletal Radiol. 2011;Jun;40(6):725-35. doi:10.1007/s00256-010-1058-2.

7. Pan Y, Li Z, Xie T, Chu CR. Hand-held arthroscopic optical coherence tomography for in vivo highresolution imaging of articular cartilage. J Biomed Opt. 2003;8:648-54.

8. Brezinski ME. Optical Coherence Tomography: Principles and Applications. Burlington: Academic Press;; 2006.

9. Drexler W, Stamper D, Jesser C. Correlation of collagen organization with polarization sensitive imaging in cartilage: implications for osteoarthritis. J Rheumatol. 2001;28(6):1311-8.

10. Liu B, Harman M, Giattina S. Characterizing of tissue microstructure with single-detector polarizationsensitive optical coherence tomography. Appl Opt. 2006;45(18):4464-79.

11. Chu CR, Lin D, Geisler JL, Chu CT, Fu FH, Pan Y. Arthroscopic microscopy of articular cartilage using optical coherence tomography. Am J Sports Med. 2004;32:699-709.

12. Han CW, Chu CR, Adachi N, Usas A, Fu FH, Huard J. Analysis of rabbit articular cartilage repair after chondrocyte implantation using optical coherence tomography. Osteoarthritis Cartilage. 2003;11:111-21.

13. Herrmann JM, Pitris C, Bouma BE, Boppart SA, Jesser CA, Stamper DL. High resolution imaging of normal and osteoarthritic cartilage with optical coherence tomography. J Rheumatol. 1999;26:62735 .

14. Chu CR, Williams A, Tolliver D, Kwoh CK, Bruno S, Irrgang JJ. Clinical Optical Coherence Tomography of Early Articular Cartilage Degeneration in Patients with Degenerative Meniscal Tears. Arthritis Rheumatism. 2010;62(5):1412-20. doi 10.1002/art.27378.

15. Zheng K, Martin SD, Rashidifard CH, Liu B, Brezinski ME. In vivo micron-scale arthroscopic imaging of human knee osteoarthritis with optical coherence tomography: comparison with magnetic resonance imaging and arthroscopy. American Journal of Orthopedics. 2010;39(3):122-5.

16. Bear DM, Williams A, Chu CT, Coyle CH, Chu CR. Optical coherence tomography grading correlates with MRI T2 mapping and extracellular matrix content. Journal of Orthopaedic Research. 28(4):546552.

17. Patel NA, Zoeller J, Stamper DL, Fujimoto JG, Brezinski ME. 2005. Monitoring osteoarthritis in the rat model using optical coherence tomography. Med Imag. 2005;24(2):155-159. 
18. Roberts MJ, Adams SB Jr, Patel NA. A new approach for assessing early osteoarthritis in the rat. Anal Bioanal Chem. 2003;377(6):1003-6.

19. Mosher TJ, Dardzinski BJ. Cartilage MRI T2 relaxation time mapping: overview and applications. Semin Musculoskelet Radiol Dec. 2004;8(4):355-68.

20. Watrin-Pinzano A, Ruaud JP, Cheli Y, Gonord P, Grossin L, Gillet P, Blum A, Payan E, Olivier P, Guillot G, Netter P, Loeuille D. T2 mapping: an efficient MR quantitative technique to evaluate spontaneous cartilage repair in rat patella. OsteoArthritis Cartilage. 2004;12:191-200.

21. Li X, Martin S, Pitris C. High-resolution optical coherence tomography imaging of osteoarthritic cartilage during open knee surgery. Arthritis Res Ther. 2005;7(2):R318-23.

\section{Figures}
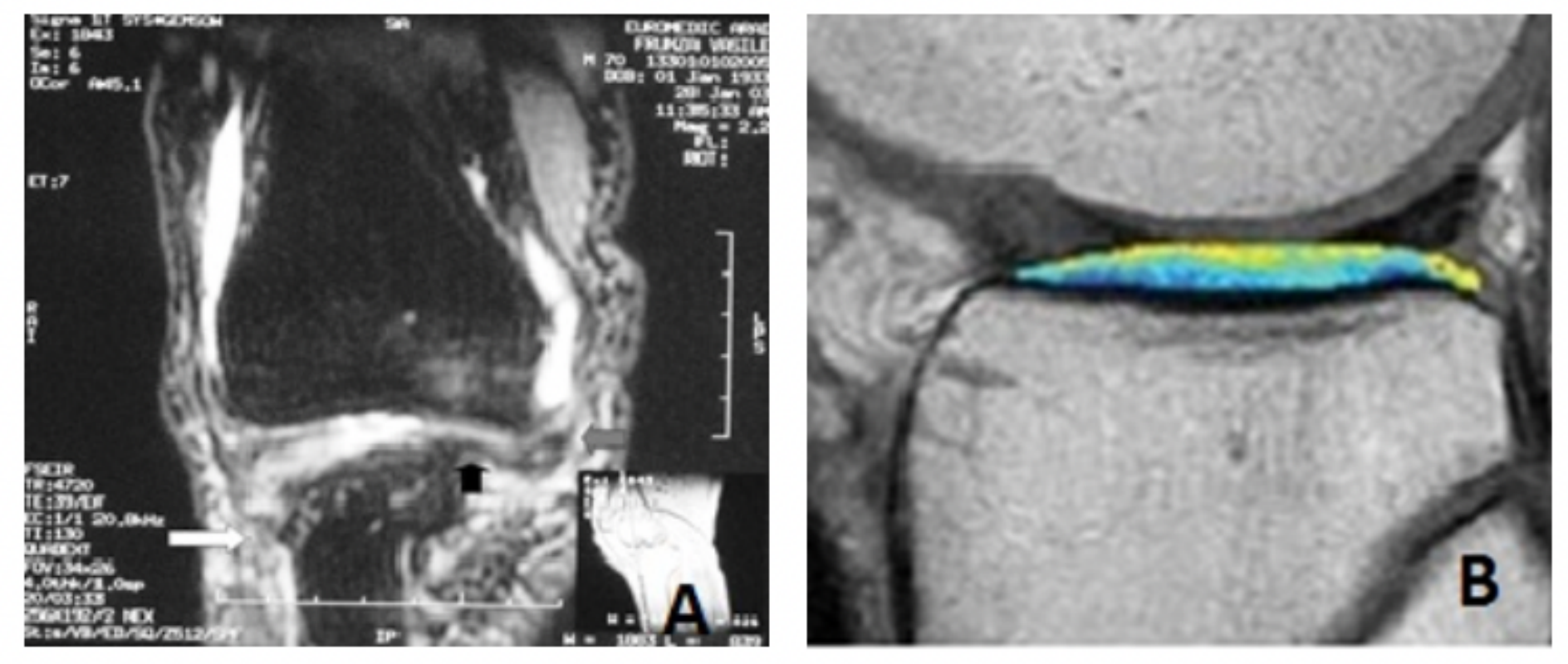

\section{Figure 1}

a) Conventional MRI Arthritic changes in the medial femoro-tibial compartment; b) Same patient lateral tibial plateau MRI T2 mapping technique; 


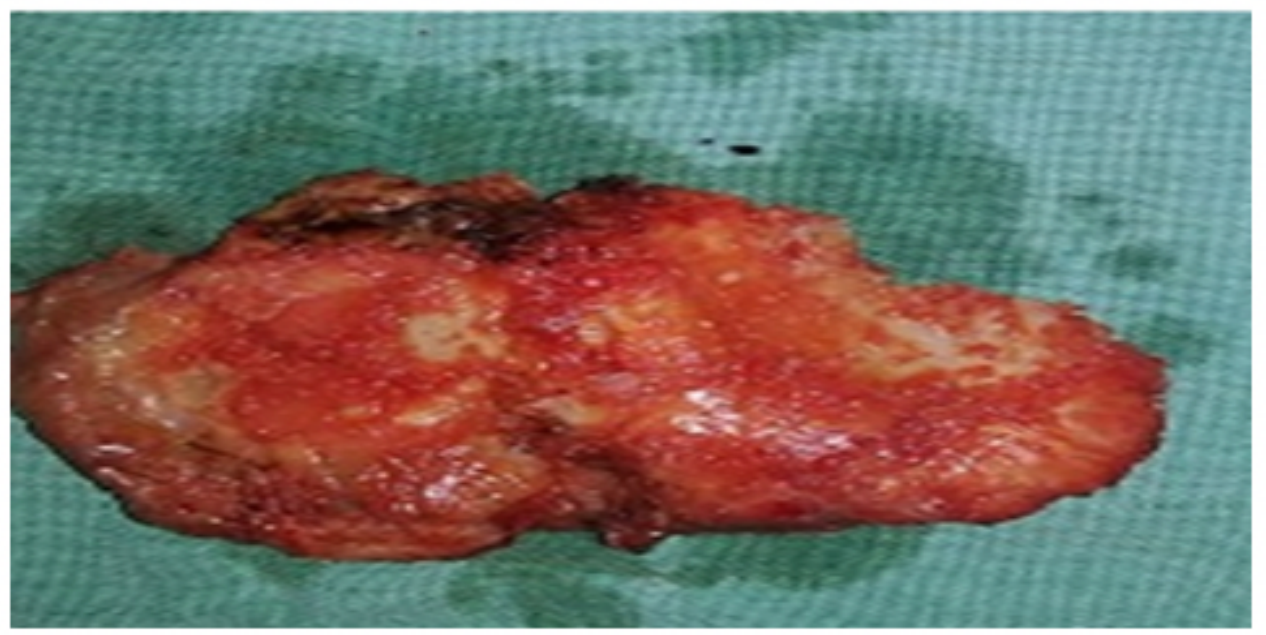

Figure 2

Anatomical specimen. Proximal tibial specimen after tibial osteotomy following total knee arthroplasty

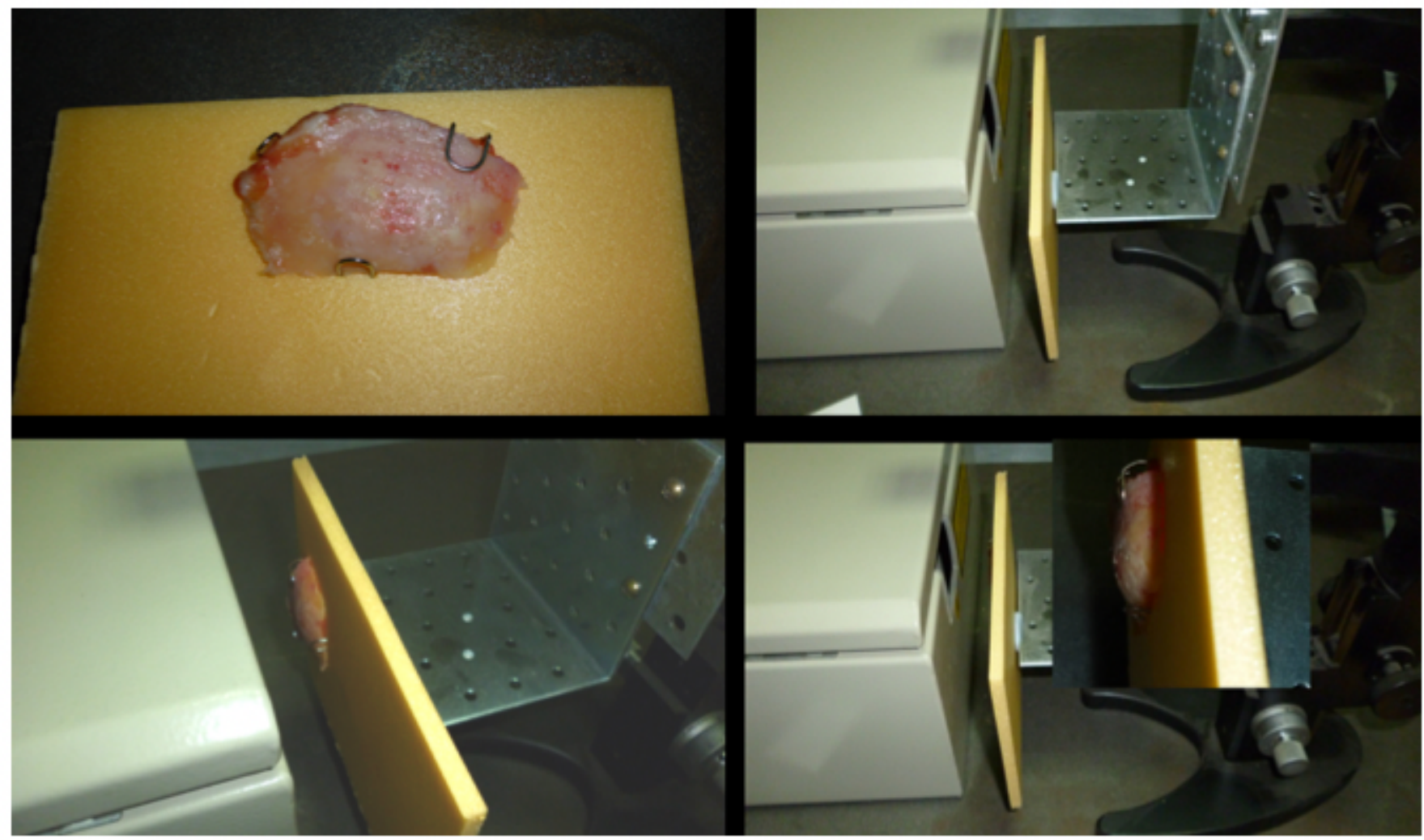

Figure 3

OCT examination of the anatomical tibial specimen 

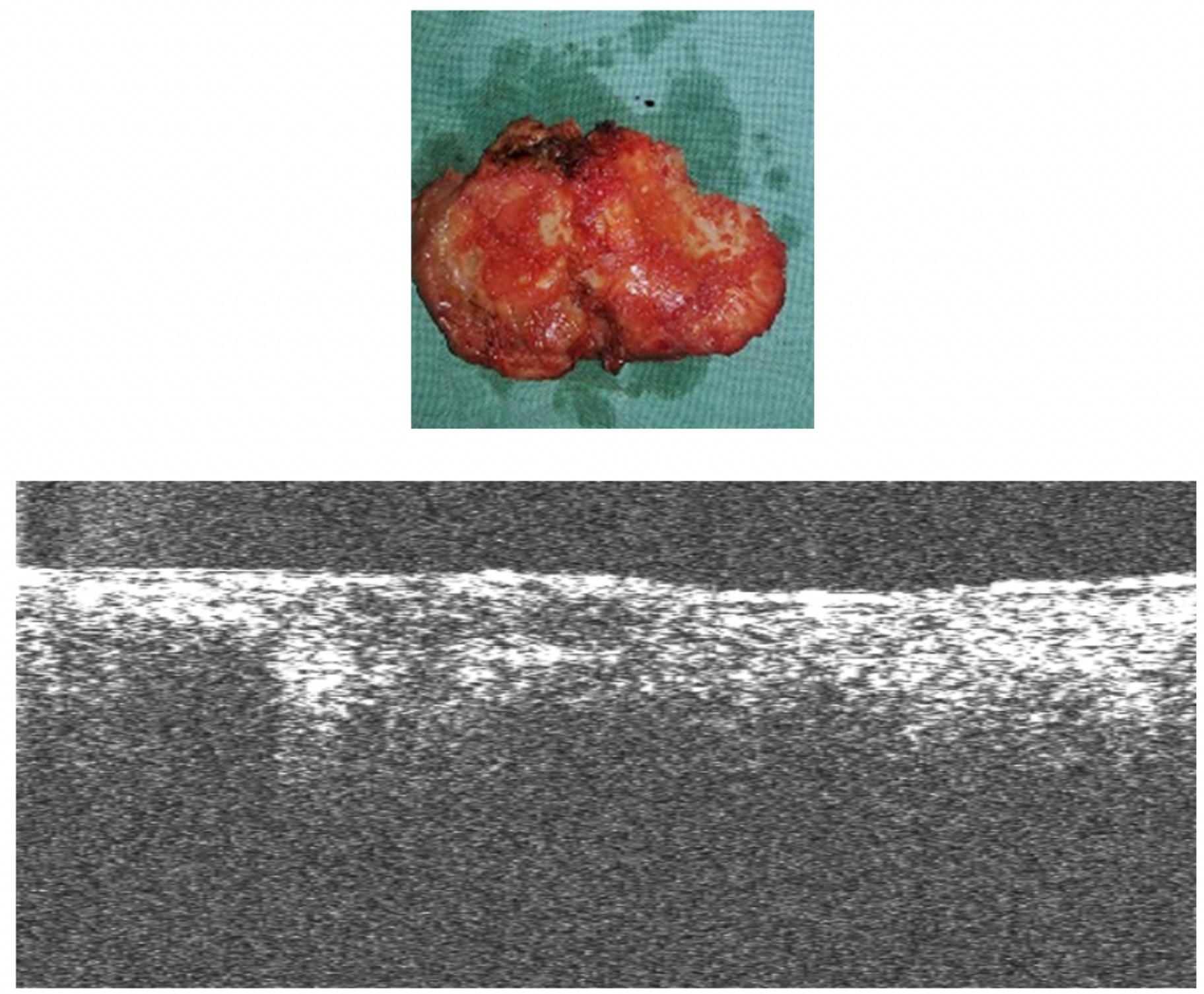

Figure 4

Patient GC, 52 years abnormal OCT cartilage image, loss of laminar structure of the collagen matrix and surface irregularities (grade2). 